\title{
Vulvar melanoma: relevant aspects in therapeutic management ${ }^{*}$
}

\author{
Adriana Bittencourt Campaner ${ }^{1}$ \\ Fernanda de Araujo Cardoso ${ }^{1}$
}

\author{
Gustavo Leme Fernandes ${ }^{1}$ \\ John Verrinder Veasey ${ }^{2}$
}

DOI: http:/ / dx.doi.org/10.1590/abd1806-4841.20174941

\begin{abstract}
Cancer of the vulva accounts for at least 1\% of malignant neoplasms among women. Although rare, vulvar melanoma is the second most common histological type of vulvar cancer, representing 7-10\% of all malignant vulvar neoplasms. Initial symptoms are non-specific and complete excision of the lesion is indicated in cases with suspected diagnosis. Prognosis of patients with these neoplasms is poor and remains unchanged despite the treatment approach. Hemivulvectomy with lymph node dissection is the current procedure of choice, associated or not with adjuvant therapies. We report two cases of patients presenting with late diagnosed vulvar melanoma and the relevant aspects in their therapeutic management.
\end{abstract}

Keywords: Histology; Melanoma; Prognosis; Therapeutics; Vulvar neoplasms

\section{INTRODUCTION}

Cancer of the vulva is a rare neoplasm, accounting for less than $1 \%$ of malignant neoplasms among women. It represents $3-5 \%$ of malignant neoplasms of the female genital tract and has an estimated incidence of 1-2 cases per 100,000 women/year. ${ }^{1}$ The most frequent histological type is epidermoid carcinoma, responsible for over $90 \%$ of cases of cancer of this organ, followed by melanoma (5-6\%). Sarcoma, basal cell carcinoma, and vulvar Paget's disease account for less than $2 \%$ of cases. ${ }^{1,2}$ With regard to location, the labia majora is the most commonly affected site $(52 \%)$ followed by the labia minora (18\%), clitoris (10-15\%) and Bartholin's glands (1-3\%).,3

Although rare, vulvar melanoma is the second most common histological type of vulvar cancer, representing $7-10 \%$ of all malignant vulval neoplasms. ${ }^{5}$ However, gynecologists and dermatologists may relatively often encounter this neoplasm, particularly within referral centers. Given that lesion size is a determinant for prognosis, early detection of the tumor is of vital importance.,

Two cases of advanced vulvar melanoma in patients treated at the pelvic oncology center of a college hospital are reported below.

\section{CASES REPORT}

Case 1

A 50-year-old patient sought the hospital due to a complaint of a 2-month history painful lesion of the vulva. The lesion, situated on the left labia minora, was painful to the touch, particularly during sexual intercourse. The patient reported an absence of any other associated gynecological or clinical symptoms. Personal history included three lesions of basal cell carcinoma in the face that had been previously treated. Concerning family history, her father presented with a melanoma on his back one year before.

Clinical examination of the vulva revealed a round tumor of $2.5 \mathrm{~cm}$ in diameter, adhering to deep planes on the inside surface of the left labium minus, exhibiting phlogistic signs suggestive of abscess. A dark patch was found adjacent to this lesion, also on the labium minus, measuring approximately $10 \times 4 \mathrm{~mm}$, which was flat and had poorly defined margins (Figure 1). Speculum exam, oncotic colpocytology, colposcopy, and digital rectal exams were all normal. Inguinal lymph nodes were not palpable.

The lesion was submitted to excisional biopsy and pathology showed a melanoma in situ, Clark I, with horizontal growth, slight lymphocitic infiltration, absence of angiolymphatic or perineural invasion, and exiguous surgical margins. A left hemivulvectomy with ipsilateral superficial lymphadenectomy was carried out. Definitive histological examination showed an acral lentiginous melanoma in the vertical growth phase, Clark IV, and Breslow $6.5 \mathrm{~mm}$. It also revealed the presence of ulceration, mitotic index of $5 / \mathrm{mm}^{2}$, with slight peritumoral and intratumoral lymphocytic infiltration, absence of regression, presence of angiolymphatic and perineural invasion, absence of satellitosis, and surgical margins free of neoplasm.

Frozen-section evaluation of sentinel lymph node tested negative, with $1 / 5$ lymph nodes affected on the assessment using the definitive immunohistochemistry assay (MELAN A).

Histologic examination was confirmed by immunohistochemistry assay, which revealed immunoexpression of S100 protein

Received on 17.07.2015

Approved by the Advisory Board and accepted for publication on 12.03.2016

* Work performed at the Hospital da Irmandade da Santa Casa de Misericórdia de São Paulo - São Paulo (SP), Brazil.

Financial support: none.

Conflict of interest: none.

Department of Gynecology and Obstetrics at Irmandade da Santa Casa de Misericórdia de São Paulo - São Paulo (SP), Brazil.

Dermatology Clinic at Irmandade da Santa Casa de Misericórdia de São Paulo - São Paulo (SP), Brazil.

(C)2017 by Anais Brasileiros de Dermatologia 
and HMB45 (CK7 negative), confirming the diagnosis of melanoma. The patient was referred to the Oncologic Dermatology outpatient unit where immunotherapy with Interferon-alpha (planned for two years) was indicated, a treatment currently underway. There have been no signs of local recurrences or distal metastases after a 7-month follow-up.

\section{Case 2}

A 58-year-old patient with a complaint of right vulvar le-

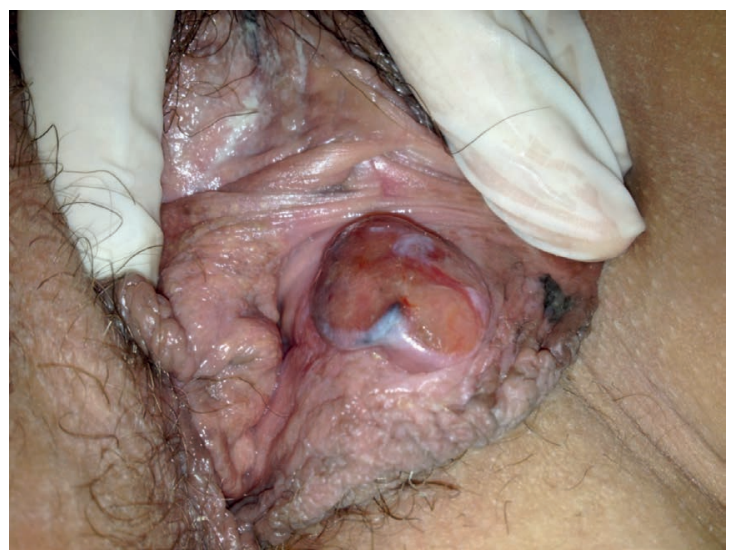

Figure 1: Case 1 - Round tumor, $2.5 \mathrm{~cm}$ in diameter, adhering to deep planes on the internal surface of the left labium minus, with central ulceration and some dark pigmented areas

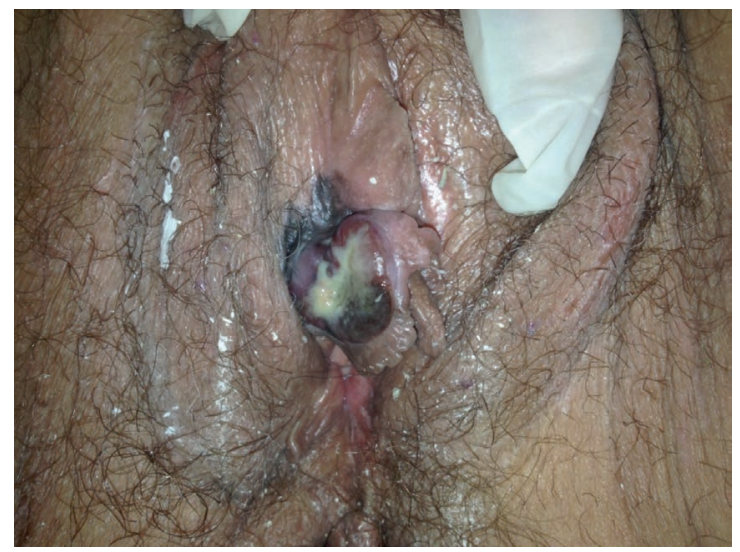

Figure 2: Case 2 - A flat pigmented lesion on the right labium minus measuring approximately $4 \mathrm{~cm}$ in diameter; a raised, vegetative reddish area can be seen on the medial face of the tumor
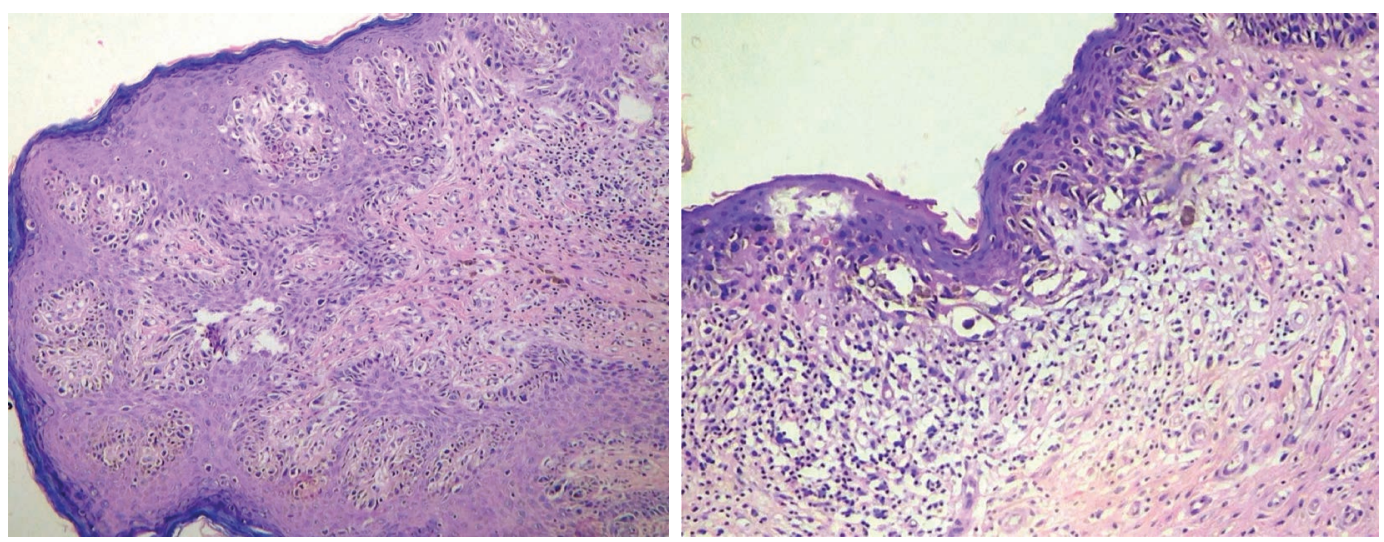

Figure 3:

Malignant melanoma of the vulva with vertical growth. (Hematoxylin \& eosin x100, Hematoxylin $\& \operatorname{eosin} \times 200)$ sion concomitant with pruritus and a 2-month history of progressive growth, in addition to discharge of foul yellowish secretion. The patient denied any other associated clinical or gynecological symptoms. She denied personal or family history of cancers. Vulvar inspection revealed a flat darkened lesion on the right labium minus, with irregular borders measuring approximately $4 \mathrm{~cm}$; there was a raised reddish vegetation on the medial aspect of the main lesion, with a drainage point of yellowish fetid secretion (Figure 2). Speculum examination, oncotic colpocytology, colposcopy, and digital rectal exams were all normal. Inguinal palpation revealed a mobile, painless lymphadenomegaly to the right side measuring $2 \mathrm{~cm}$. A biopsy of the lesion was performed and the anatomopathoogical results showed malignant melanoma of the vulva with vertical growth. The patient was submitted to right hemivulvectomy with superficial and deep bilateral lymphadenectomy. Histological amination confirmed the diagnosis of a malignant melanoma of the vulva, measuring $2.6 \times 5 \mathrm{~cm}$ (depth of $2 \mathrm{~cm}$ ), with nodular pattern, Clark IV, Breslow 20.5mm, vertical growth, mitotic index of 14/10 cga, moderate peritumoral lymphocytic infiltration and absent intratumoral invasion, ulceration present, satellitosis absent, tumor necrosis present, perineural and lymphatic invasion present, and no invasion; metastasis was detected in $1 / 5$ right superficial lymph nodes, while no metastases were observed in the left lymph nodes. The patient was referred for radiotherapy owing to the advanced stage of the lesion. She has not returned yet after radiotherapy treatment.

\section{DISCUSSION}

in order of incidence, are mucosal lentiginous (27-57\%), nodular (22$28 \%$ ), unclassified (12-16\%), and superficial spreading (4-56\%). ${ }^{5}$

Complete excision of the lesion is indicated in cases with suspected diagnosis. Currently, surgery still remains the best option albeit without the need for radical procedures. A number of studies have shown that radical surgery does not increase patient's survival with initial disease compared to local excision with margins, and is associated with greater morbidity and anatomical disfiguring. my is becoming a questionable and obsolete treatment approach. Alternatively, wide local excision with a $1 \mathrm{~cm}$ surgical margin is recareas of regression (Figure 3). All margins were free of neoplastic Consequently, radical vulvectomy with bilateral lymphadenecto- 
ommended for lesions with a depth of less than $1 \mathrm{~mm}$ and en bloc resection for deeper lesions, with a safety margin of 2-3 cm and regional lymphadenectomy (inguinal-femoral). ${ }^{6}$ Adjuvant treatment with immunotherapy, chemotherapy, and radiotherapy may be recommended in specific cases and for local recurrences and distal metastases. $^{7}$

The main prognostic factors include tumor site, depth of invasion, presence of ulceration, and occurrence of lymph node metastases. In addition, patient's age and tumor stage also appear to be critical factors in the survival of women with vulvar melanoma. ${ }^{8}$ Irrespective of stage, the literature shows that 5-year survival rates are around $20-56 \% .^{9}$ The behavior of primary malignant vulvar and extragenital cutaneous melanomas appear to be similar. However, some studies have shown that the general prognosis of patients with vulvar melanoma is worse than that of women with extragenital melanoma and squamous cell carcinoma of the vulva, showing a greater tendency for local and distal recurrence., ${ }^{710}$

Although rare, vulvar melanoma can be relatively often encountered in Oncology referral centers. The condition must be diagnosed as early as possible in order to improve the prognosis and survival of patients. $\square$

\section{REFERENCES}

1. Sturgeon SR, Brinton LA, Devesa SS, Kurman RJ. In situ and invasive vulvar cancer incidence trends. Am J Obstet Gynecol. 1992;166:1482-5.

2. Chhabra S, Bhavani M, Deshpande A. Trends of vulvar cancer. J Obstet Gynaecol. 2014;34:165-8.

3. Stroup AM, Harlan LC, Trimble EL. Demographic, clinical, and treatment trends among women diagnosed with vulvar cancer in the United States. Gynecol Oncol. 2008; 108:577-83.

4. Murzaku EC, Penn LA, Hale CS, Pomeranz MK, Polsky D. Vulvar nevi, melanosis, and melanoma: An epidemiologic, clinical, and histopathologic review. J Am Acad Dermatol. 2014:71:1241-9.

5. Trone JC, Guy JB, Mery B, Langrand Escure J, Lahmar R, Moncharmont C, et al. Melanomas of the female genital tract: state of the art. Bull Cancer. 2014;101:102-6.

6. Jahnke A, Makovitzky J, Briese V. Primary melanoma of the female genital system: a report of 10 cases and review of the literature. Anticancer Res. 2005;25:1567-74.

7. Janco JM, Markovic SN, Weaver AL, Cliby WA. Vulvar and vaginal melanoma: case series and review of current management options including neoadjuvant chemotherapy. Gynecol Oncol. 2013;129:533-7.

8. Singh S, Singh P, Kaur N. Vulvar malignant melanoma: a rare tumor with worse prognosis. Int J Reprod Contracept Obstet Gynecol. 2013;2:494-6.

9. Bradgate MG, Rollason TP, McConkey CC, Powell J. Malignant melanoma of the vulva: a clinicopathological study of 50 women. Br J Obstet Gynaecol. 1990;97:124-33.

10. Suwandinata FS, Bohle RM, Omwandho CA, Tinneberg HR, Gruessner SE. Management of vulvar melanoma and review of the literature. Eur J Gynaecol Oncol. 2007;28:220-4.

\author{
MAILING ADDRESS: \\ John Verrinder Veasey \\ Rua Dr. Cesário Mota Jr, 112 - Vila Buarque \\ 01221-020 - São Paulo - SP \\ Brazil \\ E-mail: johnveasey@uol.com.br
}

How to cite this article: Campaner AB, Fernandes GL, Cardoso FA, Veasey JV. Vulvar melanoma: relevant aspects in therapeutic management. An Bras Dermatol. 2017;92(3):398-400. 